\title{
Propylene glycol produces excessive apoptosis in the developing mouse brain, alone and in combination with phenobarbital
}

\author{
Karen Lau', Brant S. Swiney', Nick Reeves', Kevin K. Noguchi' and Nuri B. Farber ${ }^{1}$
}

INTRODUCTION: Propylene glycol (PG) is a common solvent used in medical preparations. It is generally recognized as safe at regulated concentrations; however, its apoptotic potential is unknown.

RESULTS: PG triggered widespread apoptotic neurodegeneration with the greatest damage at postnatal day 7 (P7). Significant apoptosis was observed at doses as low as $2 \mathrm{ml} / \mathrm{kg}$. These findings have implications for the safety of drug preparations used in pediatric medicine. The anticonvulsant phenobarbital (PB), which alone produces apoptosis in the immature central nervous system (CNS) is prepared in 68\% PG and 10\% ethanol $(\mathrm{EtOH})$. We assessed whether PG contributes to the neurotoxic potential of PB. The agents (both at subtoxic doses) produce significantly more apoptosis when used in combination.

DISCUSSION: In conclusion, finding an alternative nonapoptotic solvent that can be used as a substitute for PG may be beneficial to patients.

METHODS: C57BL/6 mice (P4-30) were exposed to PG to examine whether PG could produce apoptosis in the developing CNS.

A poptosis is a genetically regulated endogenous cellular process in which a variety of extrinsic and intrinsic signals can activate multiple independent pathways resulting in cell death (1). Ethanol (EtOH), a commonly abused solvent, produces widespread apoptotic neurodegeneration in the developing mammalian brain $(2,3)$. Recently, we reported that dimethyl sulfoxide (DMSO), a widely used solvent in industry and biological research that is routinely given to children as part of a transplantation procedure, is able to mimic this neurodegenerative process in the developing mouse brain (4). This solvent-induced apoptosis appears to be most severe during the period of synaptogenesis (the brain growth spurt). In rodents, the period of vulnerability occurs during the first several weeks of postnatal life, whereas in humans, it extends from the second trimester of gestation to several years after birth (5).

Propylene glycol (PG; 1, 2-propanediol) is another solvent frequently used in the preparation of pharmaceuticals, as well as in foods and cosmetics. The US Food and Drug Administration has identified PG as being "generally recognized as safe" (6), with an acceptable daily intake of up to $25 \mathrm{mg} / \mathrm{kg}$ (7). Although it is considered to be a nontoxic "inactive" ingredient in pharmaceuticals, the American Academy of Pediatrics Committee on Drugs recognized PG as a potential toxicant over 20 years ago (8). These concerns developed as a result of early studies linking PG to central nervous system (CNS) disturbances in children, including depression and seizures $(9,10)$. During the last decade, PG toxicity has been increasingly reported in human infants and adults. Adverse effects associated with PG exposure include hyperosmolality, metabolic abnormalities, hypotension, renal dysfunction, and multiorgan failure (11-18); however, there are no recent studies directly linking PG exposures to CNS disturbances in humans.

Many agents used in pediatric neurology are prepared in a liquid medium containing 30 to $80 \%$ PG, and often also containing $10 \% \mathrm{EtOH}$. The use of $\mathrm{PG}$ as a vehicle for i.v. drugs is of major concern for two reasons. First, infants can become exposed to extremely high doses of PG, as is the case in the pediatric intensive care unit, where sedatives are administered as long-term i.v. infusions $(19,20)$. Second, many clinically used agents themselves can produce apoptosis in the immature CNS, including phenobarbital (PB) $(21,22)$, which is prepared in $68 \%$ (v/v) PG and $10 \% \mathrm{EtOH}$. The combined effects of chemicals in these mixtures are unclear and have not been widely studied. Collectively, they may have some adverse effects that are greater than expected, even if their individual doses are subtoxic.

Little information is available about the direct neurotoxic potential of PG. The purpose of this study was therefore to examine whether PG could induce apoptosis in the developing CNS. Since PG is used as the vehicle for compounds that are known to produce apoptosis, we also assessed whether the combined effect of PB dissolved in PG would produce significantly more neuroapoptosis than $\mathrm{PB}$ dissolved in saline.

\section{RESULTS}

Pattern and Time Course of Caspase-3 Activation

Caspase-3 is the major effector caspase in the CNS (23), and immunohistochemical detection of activated caspase-3 (AC-3) immunoreactivity has proven to be a sensitive measure of 
apoptosis (3). Brains were initially examined at $8 \mathrm{~h}$ after injection because this time point was found to produce the peak levels of damage of intact cells in our previous studies (4). In saline brains, AC-3-positive neurons were sparse and sporadically distributed within various regions, showing a pattern typical of physiological cell death (Figure 1). PG produced a striking increase in AC-3-positive cells throughout the brain (Figure 1), as compared to saline-treated animals. The most severely affected areas were the caudate/putamen $(\mathrm{CPu})$ and cortical regions (especially the retrosplenial, cingulate, and motor cortex). The nucleus accumbens, the superior colliculus, the pyramidal cell layer of the hippocampus, the subiculum, and specific areas of the thalamus (lateral dorsal (LD Th), lateral rostral, reticular, lateral geniculate nucleus) were also damaged. Affected cells were relatively intact in most of these regions, with staining in the nucleus, cell body, and entire dendritic tree. However, staining in the $\mathrm{CPu}$ was localized to cell bodies (Figure 1f) and cells appeared to be in an advanced apoptotic stage, suggesting that the onset of degeneration in this region begins at an earlier time point or that degeneration progresses at a faster rate. Furthermore, damage appeared to be more intense in the lateral part of the $\mathrm{CPu}$.

The pattern of degeneration was then examined following other survival intervals: $2 \mathrm{~h}, 4 \mathrm{~h}, 12 \mathrm{~h}$, and $24 \mathrm{~h}$ after injection $(n=24)$. These time-course experiments (Figure 2$)$ revealed that the process of cell death was relatively rapid, with cells beginning to degenerate as early as $2 \mathrm{~h}$ (Figure $2 \mathrm{e}-\mathbf{h}$ ). At $2 \mathrm{~h}$, the majority of damage was concentrated in the $\mathrm{CPu}$ (Figure 2f); however, there was sporadic scattering of AC-3positive cells over most cortical regions, with more intense damage to layer 2 neurons. Pyramidal cells in the ventral aspects of the hippocampus were also damaged. There was an overall increase in apoptotic cell death at $4 \mathrm{~h}$ (Figure $2 \mathrm{i}-1$ ), at which point dendrite staining was visible. Peak levels of damage occurred at $8 \mathrm{~h}$ (Figure $2 \mathrm{~m}-\mathrm{p}$ ). Sporadic damage was still present; however, damage to specific regions was more obvious, and cortical damage had progressed to involve layer 4 neurons. At $12 \mathrm{~h}$, overall AC-3 staining was decreased (Figure $2 \mathrm{q}-\mathrm{t}$ ). At $24 \mathrm{~h}$, staining of intact AC-3-positive cells in affected brain regions was dramatically reduced. In most of these animals, the AC-3 levels were similar to those seen in saline controls. However, the most severely affected animal after $24 \mathrm{~h}$ of treatment is shown in Figure $2 \mathbf{u}-\mathbf{x}$, where AC-3positive cells are clearly elevated from controls. Furthermore, an extensively diffuse non-specific type of staining was present in these regions at $24 \mathrm{~h}$, possibly owing to the presence of cellular debris. de Olmos silver staining at 24 h shows severely fragmented cells that display the characteristics of late-stage apoptosis, including condensed cell bodies and compact debris of the degeneration products (Figure 3a). To confirm whether PG produced degeneration through an apoptotic mechanism, electron microscopy was used. Cell death triggered by PG was found to have morphological characteristics of apoptosis $(24,25)$, including chromatin balls, cytoplasmic condensation, and disintegration of the nuclear membrane (Figure 3b-c).

\section{Dose Dependency of PG-Induced Apoptosis}

PG produces apoptotic neurodegeneration in a dose-dependent manner $(F(5,35)=42.55, P<0.0001$; Figure 4$)$. At $1 \mathrm{ml} / \mathrm{kg}$, there was no significant difference between AC-3 cell counts for PG-treated and saline animals. However, compared to saline, significantly greater damage was observed at $2 \mathrm{ml} / \mathrm{kg}$ $(P=0.0042)$. At this dose, approximately 60,000 neurons died by apoptosis compared to background levels of 30,000 as seen in controls. At $10 \mathrm{ml} / \mathrm{kg}$, over 500,000 neurons were undergoing apoptosis. Considerable animal death occurred at doses above $10 \mathrm{ml} / \mathrm{kg}$. Consequently, complete dose-response data were not developed, and an $\mathrm{ED}_{50}$ value for $\mathrm{PG}$ could not be calculated.

\section{Assessment of the Combined Effect of PB and PG}

PB $(5 \mathrm{mg} / \mathrm{kg})$ prepared in saline produced apoptosis levels similar to those of saline controls (Figure 5). However, PB prepared in a subtoxic dose of $68 \%$ (v/v) PG (as given clinically) produced significantly more apoptosis than $\mathrm{PB}$ in saline alone $(P<0.05)$. Approximately 12,000 more neurons died when PG was used as a solvent, that is, an amount almost $50 \%$ greater than the damage observed when saline was used. As a result of concern that this observed effect between PG and PB could be a reflection of a pharmacokinetic interaction in which PG raised the concentration of $\mathrm{PB}$, we conducted additional experiments using higher doses of PB in saline. We found that although $20 \mathrm{mg} / \mathrm{kg}$ of PB in saline did not produce any detectable apoptosis, $40 \mathrm{mg} / \mathrm{kg}$ did. Thus, if a pharmacokinetic interaction were to underlie the observed effect, PG would need to produce a four- to eightfold increase in the concentration of $\mathrm{PB}$ in order to account for the observed effect.

\section{Age Dependency of PG-Induced Apoptosis}

Severity and location of damage varied at different ages (Figure 6). The greatest amount of PG-induced apoptosis occurred in postnatal day 7 (P7) animals (Figure 6f-j). At P4 (Figure 6a-e), the pattern of degeneration was similar to that of P7; however, the overall damage was less severe. The sporadic pattern of background staining was present, and some of the same regions were damaged including the $\mathrm{CPu}$, most cortical regions, and thalamic nuclei. However, there were some subtle differences between P4- and P7-treated animals. Some regions that were damaged at P7 did not appear to be affected at P4: dorsal lateral geniculate nucleus, superior colliculus, subiculum, auditory cortex, and visual cortex. The most striking difference was observed in the distribution of AC-3 cells within the cortex. At P4, damage was limited to layer 4 neurons, whereas at P7, there was damage to layer 2 and layer 4 neurons. At P14 (Figure 6k-o), the overall damage was dramatically reduced. Sporadic background staining was still present, and most cortical regions were damaged; however, very little damage was observed in the $\mathrm{CPu}$ or in the LD Th. Apoptosis also occurred in new regions that were not damaged at P4 and P7, the most noticeable area being the granule cell layer of the dentate gyrus (Figure $6 \mathrm{~m}$ ). In addition, the neuroepithelium, anterior olfactory nucleus, and dorsal 


\section{Articles | Lau et al.}

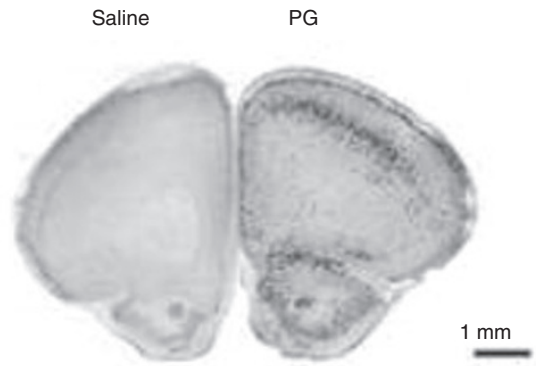

Rostro-caudal level: 2.19 mm

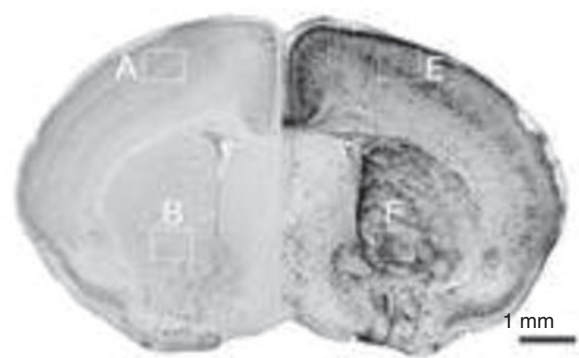

Rostro-caudal level: $3.39 \mathrm{~mm}$

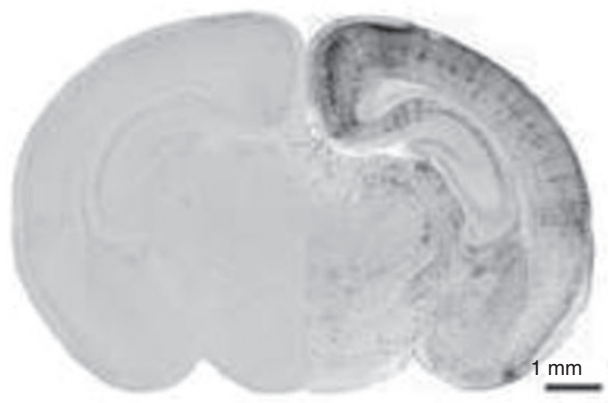

Rostro-caudal level: $5.43 \mathrm{~mm}$

Motor cortex

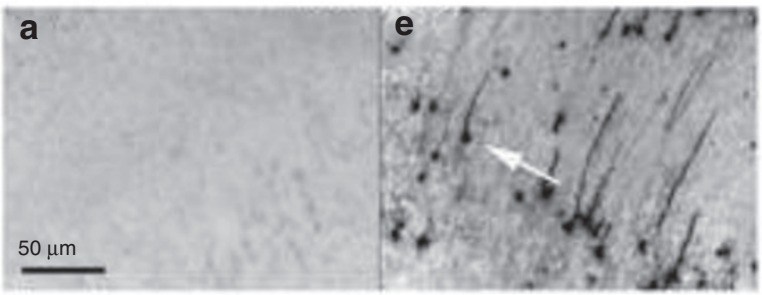

Caudate/putamen

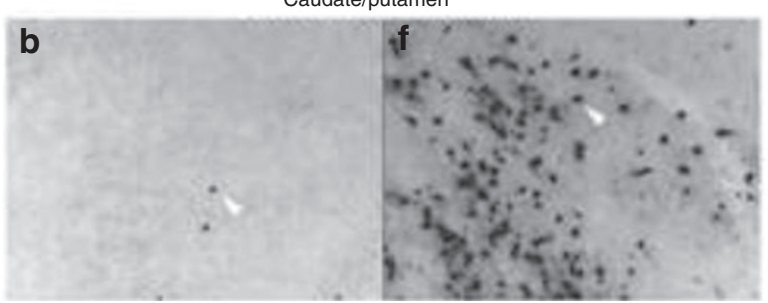

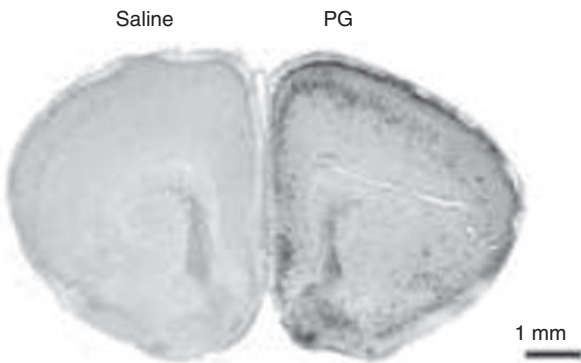

Rostro-caudal level: $2.55 \mathrm{~mm}$

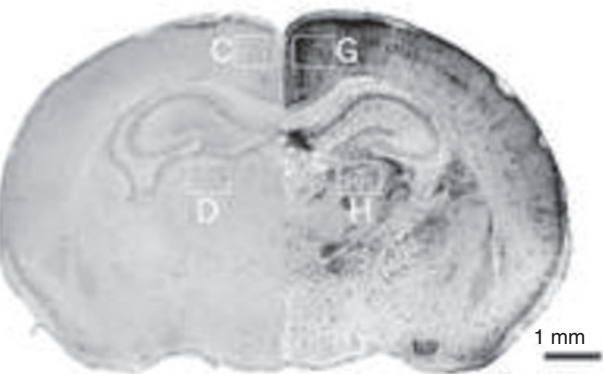

Rostro-caudal level: $4.50 \mathrm{~mm}$

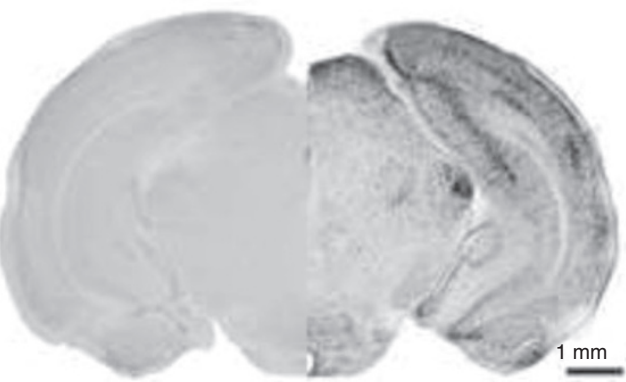

Rostro-caudal level: $6.27 \mathrm{~mm}$
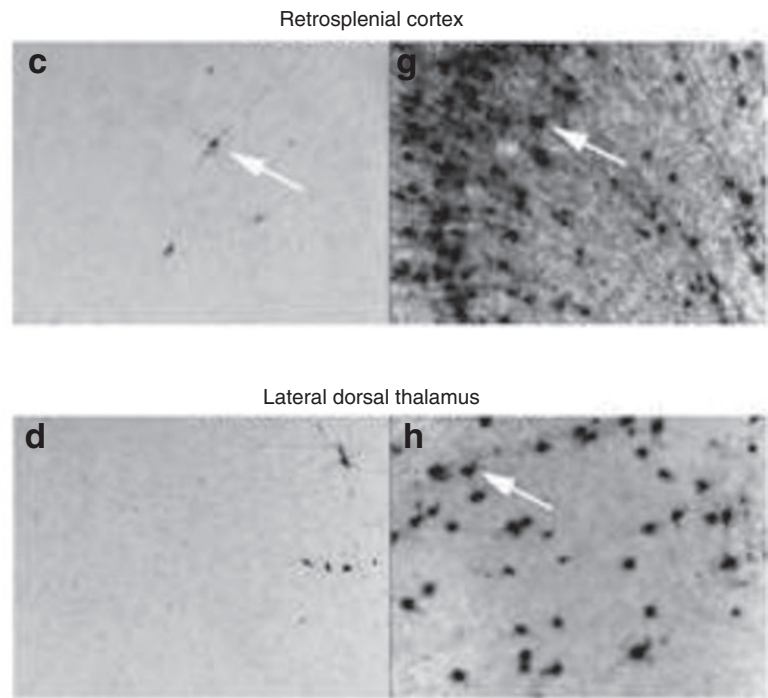

Figure 1. Sections from postnatal day 7 (P7) mouse brain following 8-h treatment with saline or propylene glycol (PG) stained immunohistochemically with antibodies to activated caspase-3 (AC-3). Saline brains (a-d) showed a pattern of sparse AC-3-positive neurons (white arrows) that is typical of physiological cell death, whereas PG triggered widespread neuroapoptosis in many brain regions (e-h). In the caudate/putamen (CPu), cells appeared to be in a later stage of the apoptotic process (staining localized to intact condensed cell bodies, white arrowheads). Top images: original magnification $\times 10$. High-powered photomicrographs: original magnification $\times 20$. 

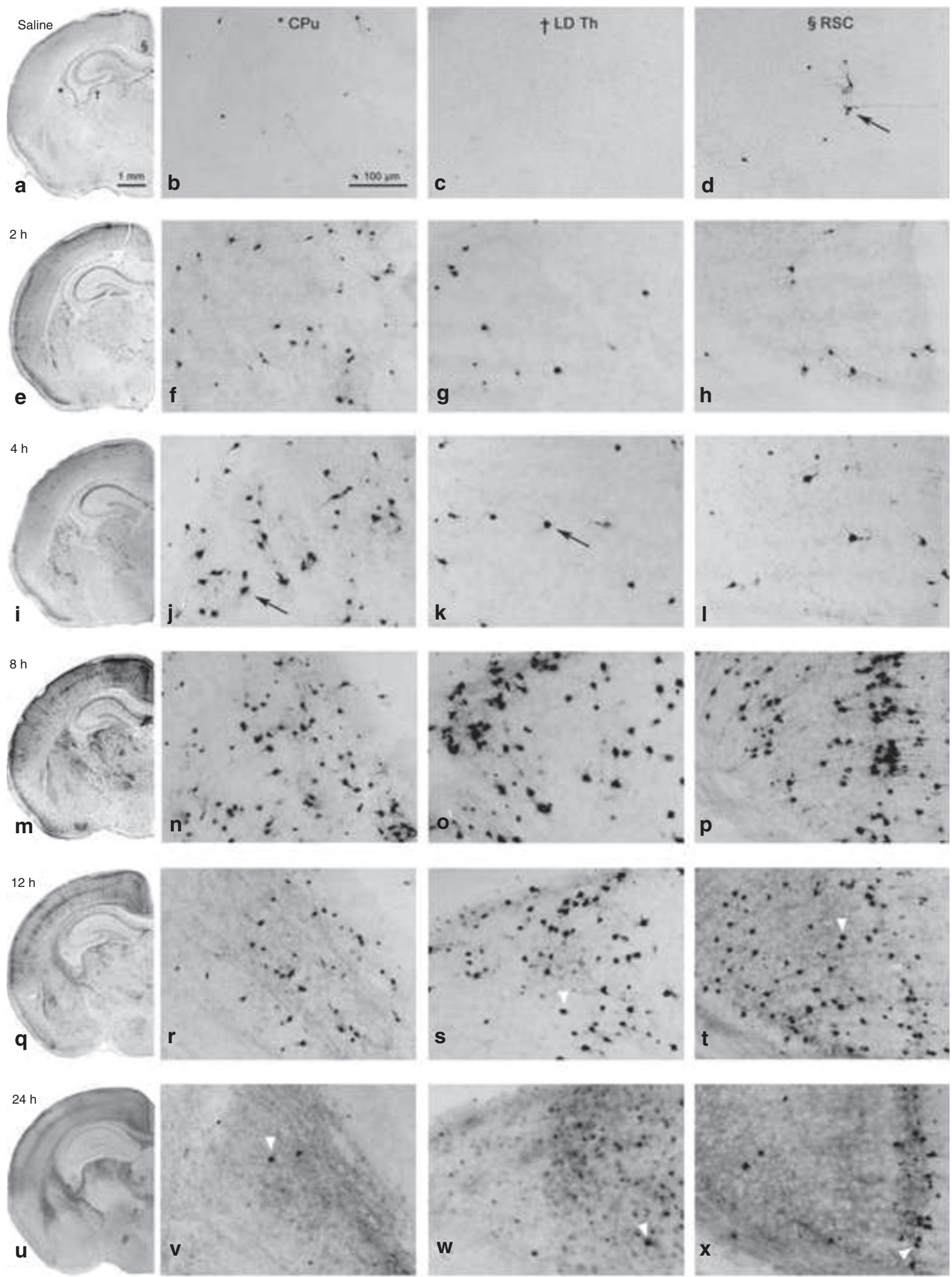

Figure 2. Histological sections from postnatal day 7 (P7) mouse brain treated with saline (a-d) or propylene glycol (PG) (e-x) at various time points after exposure. Black arrows depict activated caspase-3 (AC-3)-positive cells. Hemisections are taken from the same rostro-caudal level (original magnification $\times 10)$. High-powered photomicrographs (original magnification $\times 20)$ were taken from the ${ }^{*}$ caudate/putamen $(\mathrm{CPu}){ }^{\dagger} \mid \mathrm{lateral}$ dorsal thalamus $(\mathrm{LD} T \mathrm{Th})$, and ${ }^{5}$ retrosplenial cortex (RSC), as indicated by a symbol in (a). Onset of damage appeared at $2 \mathrm{~h}$ in the $\mathrm{CPu}(\mathbf{f})$. Peak levels of damage occurred at $8 \mathrm{~h}$ in most regions $(\mathbf{m}-\mathbf{p})$. Overall, AC-3 staining was decreased at $12 \mathrm{~h}(\mathbf{q}-\mathbf{t})$ and $24 \mathrm{~h}(\mathbf{u}-\mathbf{x})$, where staining was specific to intact cell bodies (white arrowheads). In addition to localized staining, a non-specific diffuse staining was observed at $24 \mathrm{~h}$. 


\section{Articles Lau et al.}

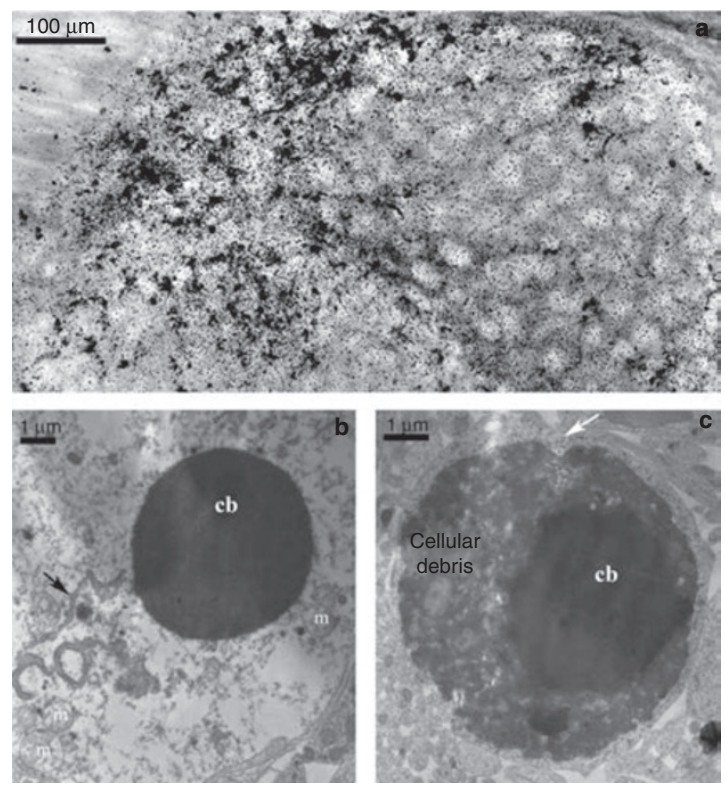

Figure 3. De Olmos silver staining at $24 \mathrm{~h}$ shows advanced-stage degeneration. Image $\mathbf{a}$ is taken from lateral dorsal thalamus (LD Th) of a postnatal day 7 (P7) animal exposed to $10 \mathrm{ml} / \mathrm{kg}$ of propylene glycol (PG) (original magnification $\times 10$ ). Cell bodies are amorphous, axons and dendrites are no longer visible, and cellular debris is scattered throughout the region. Electron microscopy photomicrographs (original magnification $\times 10,000$; b-c) demonstrate cells with classical hallmarks of apoptosis ( $8 \mathrm{~h}$ exposure to $10 \mathrm{ml} / \mathrm{kg}$ of $\mathrm{PG}$ ). Image $\mathbf{b}$ shows that the nuclear membrane is no longer intact (broken membrane remnant: black arrow), allowing nuclear and cytoplasmic compartments to mix. Mitochondria ( $\mathrm{m}$ ) appear to be swollen, and a condensed spherical chromatin ball (cb) has formed. A very late-stage apoptotic cell (c) shows migration of nuclear chromatin masses into the cytoplasm and disintegration of material inside the cell. The apoptotic cell can be distinguished from adjacent tissue cells; however, the surrounding phagocyte has already started to encroach into the degenerating cell (white arrow).

aspects of the hippocampus were also damaged at P14. At P17 (Figure 6p-t), damage was still present in the dentate gyrus (Figure 6r) and anterior olfactory nucleus. The specific layers of damage in the cortex as previously seen at P4, P7, and P14 could not be seen at this age. Damage could now be seen in the $\mathrm{CPu}$; however, closer examination of the cells showed that damaged cells appeared to be oligodendrocytes (Figure 6t). No damage was detected at P24 (Figure 6u-y) and P30 (not shown).

\section{DISCUSSION}

PG is used as a solvent for topical, oral, and injectable medications and is generally considered to be safe. However, we found that PG produces neurodegeneration in the developing mouse CNS. The degenerative process was relatively rapid, with cells beginning to fragment within $12 \mathrm{~h}$ of drug exposure. The degeneration is AC-3 positive and has morphological characteristics of apoptosis, as confirmed by electron microscopy. To our knowledge, this is the first report to show that PG produces apoptosis in the CNS of any species. The pattern of degeneration seen with PG was largely similar to those of EtOH (2) and DMSO (4). Likewise, the observed damage was dependent on age at the time of exposure. Peak sensitivity was seen at P7, when damage

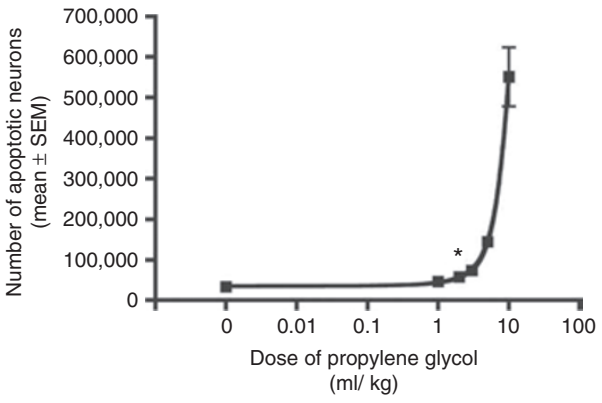

Figure 4. Postnatal day 7 (P7) animals were exposed for $8 \mathrm{~h}$ to various doses of propylene glycol (PG). Total activated caspase-3 (AC-3)-positive neurons were estimated using stereology. Number of degenerating cells increased with increasing doses of $P G(F(5,35)=42.55, P<0.0001$; $R^{2}=0.99 ; n=6$ at each dose tested). Significantly greater damage was observed at $2 \mathrm{ml} / \mathrm{kg}$ compared to saline ( ${ }^{*} P=0.0042$ ). Error bars are too small to be seen.

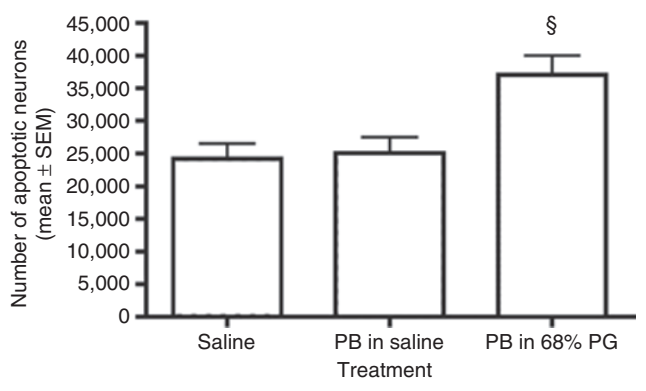

Figure 5. The combined effect of phenobarbital (PB) and propylene glycol (PG), both used at subtoxic doses. A preparation of $5 \mathrm{mg} / \mathrm{kg}$ of PB in saline $(n=8)$ produces levels of apoptosis similar to saline alone $(n=8)$. PB prepared in $68 \%$ PG $(n=10)$ produces significantly more apoptosis than PB prepared in saline $\left({ }^{5} P<0.05\right)$.

was widespread but appeared to affect specific populations of neurons. The damage was most striking in the $\mathrm{CPu}$, thalamus, and layers 2 and 4 of the cortex. The specific cell populations undergoing degeneration also varied when tested at different ages. The most noticeable difference was that damage was consistently seen in the dentate gyrus at P14 and P17, whereas it was not seen at earlier ages.

$\mathrm{EtOH}$-induced neuroapoptosis is believed to be mediated by a dual mechanism: blockade of $N$-methyl-D-aspartate (NMDA) receptors and hyperactivation of $\gamma$-aminobutyric acid-A $\left(\mathrm{GABA}_{\mathrm{A}}\right)$ receptors (2). Although the pattern of PG-induced neuroapoptosis has a close resemblance to that of $\mathrm{EtOH}$, there was no damage in the anterodorsal nucleus of the thalamus (AD). Damage in this area by $\mathrm{EtOH}$ has been attributed to its GABAergic activity. Lack of damage in the AD following PG exposure suggests that PG does not produce damage through GABA receptors. DMSO has been reported to inhibit NMDA receptors (26). NMDA antagonists produce apoptosis (27); anticonvulsants that inhibit voltage-gated sodium channels are also known to induce apoptosis (21). It is unknown whether PG has any of these properties that could account for its apoptogenic liability. Further work is needed to determine the mechanism underlying the observed damage. 


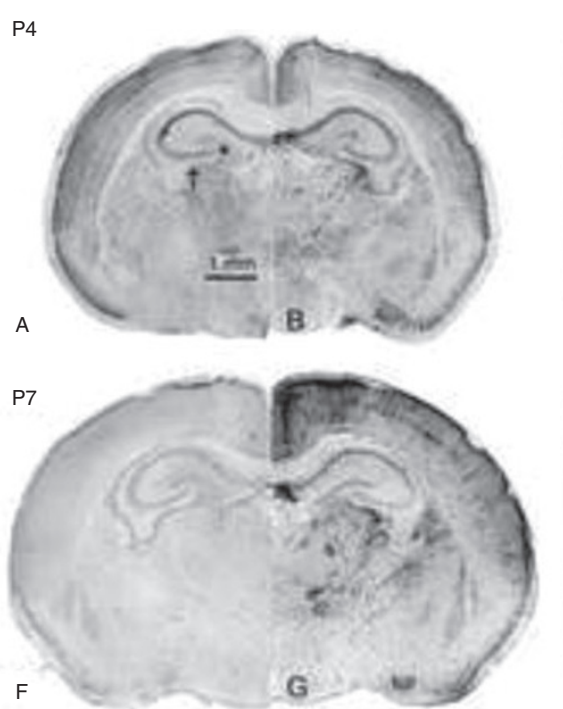

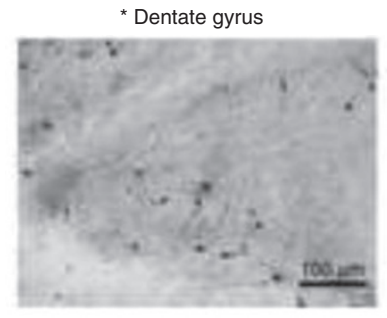

C

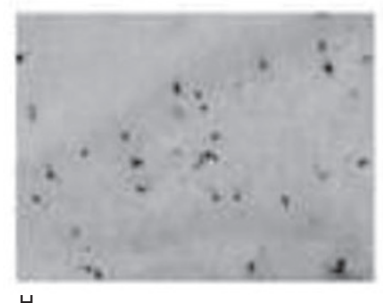

$\mathrm{H}$

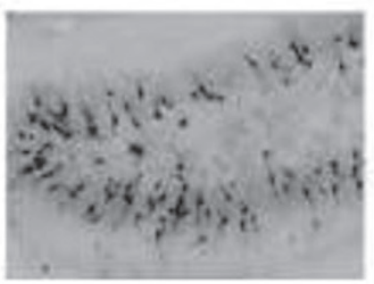

M

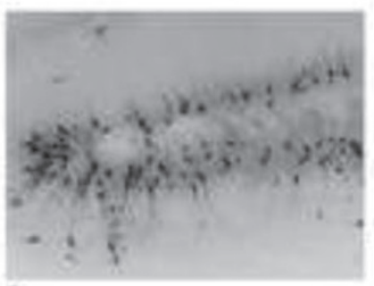

R

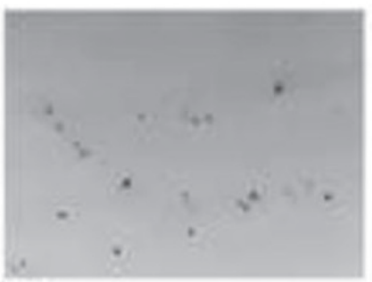

w

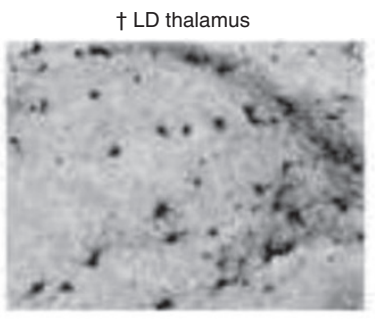

D
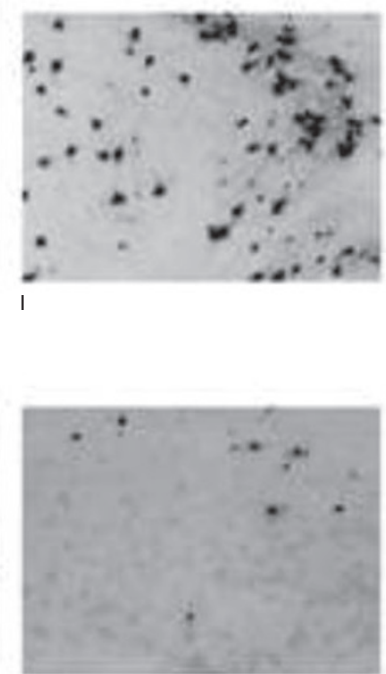

N

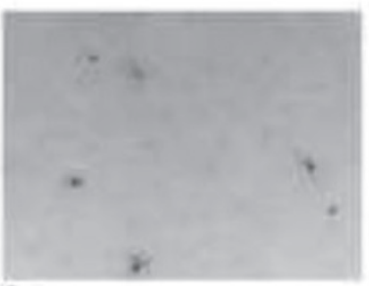

S

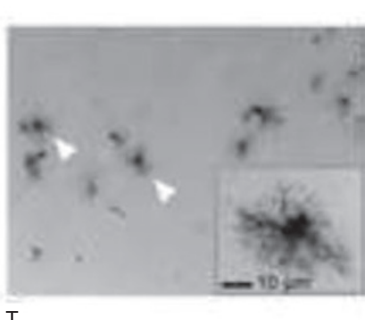

T
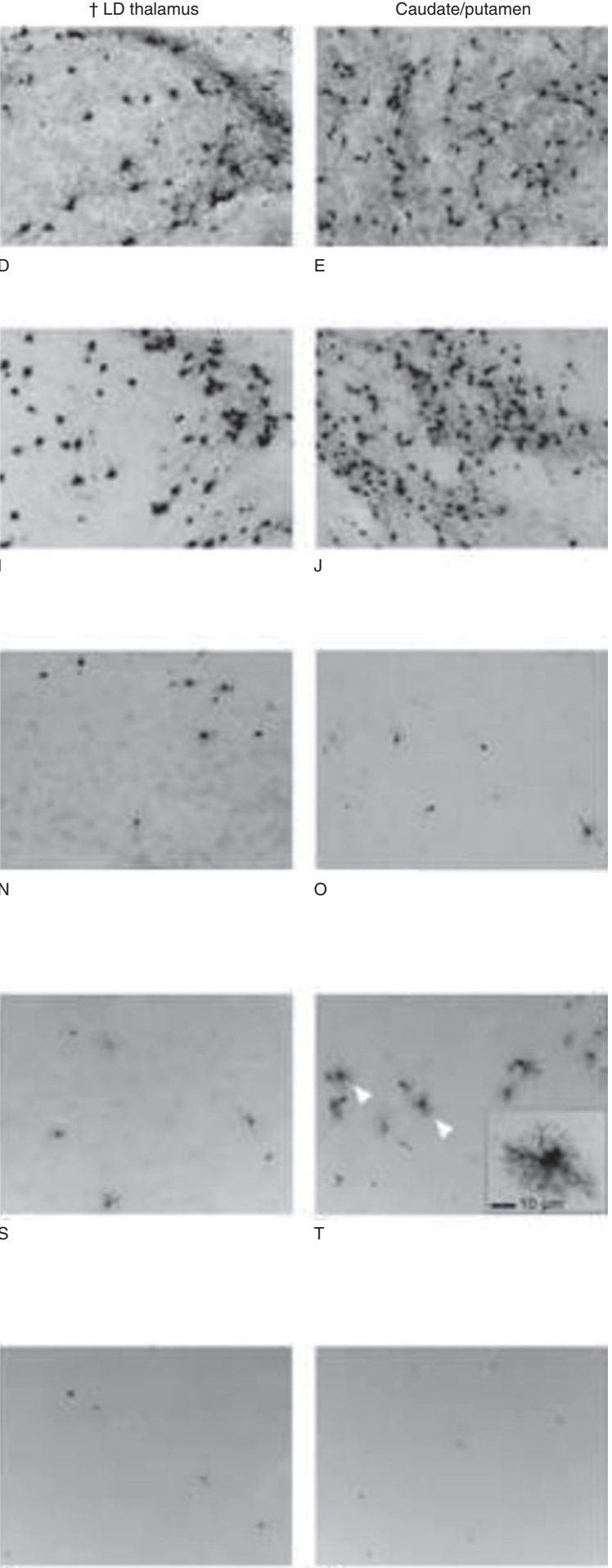

X

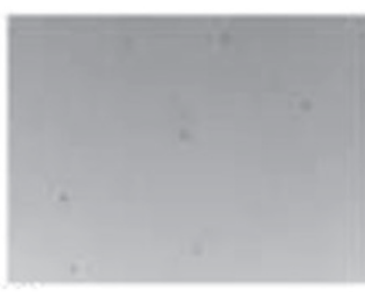

Y

Figure 6. Histological sections from P4-24 mouse brain following 8-h treatment with saline (left hemisections) or propylene glycol (PG; right hemisections) (original magnification $\times 10$ ). *Dentate gyrus and lateral dorsal thalamus (LDTh) photomicrographs are taken from the same section as those indicated by a symbol in (a). Caudate/putamen ( $\mathrm{CPu}$ ) photomicrographs were taken from a more anterior section of the brain to demonstrate the extent of damage in this region. High-powered images: original magnification $\times 20$. At P4 (a-e), severe damage was seen in the LD Th and CPu. These same regions were damaged at P7 (f-j), the age at which overall damage peaked. At P14 (k-o), overall damage was decreased, and both LD Th and CPu showed similar levels of activated caspase-3 (AC-3)-positive cells as saline. The granule cell layer of the dentate gyrus was severely damaged at P14 (m), whereas this region was not damaged at P4 (c) or P7 (h). At P17 (p-t), AC-3-positive cells were still present in the dentate gyrus (r); however, overall damage was further reduced. A new population of dying cells appeared in the CPu (white arrowheads). Closer examination of these cells showed morphology typical of oligodendrocytes ( $\mathbf{t}$; box: original magnification $\times 40)$. At P24 (u-y), damage was similar to saline. Staining was extremely sparse, making it difficult to see at low magnification. 


\section{Articles | Lau etal.}

Infants are readily exposed to PG in foods and medicines. The use of $\mathrm{PG}$ as a vehicle for pediatric i.v. medications (e.g., diazepam, lorazepam, phenytoin, and $\mathrm{PB}$ ) means that infants are exposed to PG during a critical period of neural development, when their brains would be sensitive to apoptogenic effects of the solvent. The apoptotic effect of PG was found to be dose dependent, with significant apoptosis detected at doses as low as $2 \mathrm{ml} / \mathrm{kg}$. However, the effect was not significant at $1 \mathrm{ml} / \mathrm{kg}$. Based on these results, we conclude that the toxic threshold is between 1 and $2 \mathrm{ml} / \mathrm{kg}$. The amount of PG that a child would receive following a single loading dose of $40 \mathrm{mg} / \mathrm{kg} \mathrm{PB}$ is $0.2 \mathrm{ml} / \mathrm{kg}$. A 5- to 10 -fold difference between routine clinical exposure and the toxic threshold would suggest that a one-time exposure to PG is unlikely to produce apoptosis. However, it is unknown whether humans and mice produce similar blood PG levels following the same $\mathrm{mg} / \mathrm{kg}$ dose. Because of potential interspecies differences in pharmacokinetics, the true outcome in humans is difficult to predict. Pharmacokinetic studies determining the blood PG levels achieved following PG exposure in both newborn mice and human neonates are needed to ascertain whether our initial conclusion about the safety of a single exposure is correct.

More concerning are instances of human infants being exposed to PG over a prolonged period, because the toxic threshold is likely to be surpassed. For example, significant PG accumulation in serum (ninefold increase from baseline levels to the end of infusion) has been reported in infants receiving continuous infusion of lorazepam for sedation (19). A more recent study reported that critically ill neonates who were administered medications through continuous infusion were exposed to doses of PG that were 180 times the acceptable daily intake (20). Furthermore, for treatment of certain clinical conditions such as status epilepticus, it is not uncommon to use multiple drugs, many of which are prepared as solutions containing 30-80\% PG. Typically, lorazepam (prepared in $80 \% \mathrm{PG}$ and $10 \% \mathrm{EtOH}$ ) is administered intravenously at $\sim 0.1 \mathrm{mg} / \mathrm{kg}$. If seizures are not immediately arrested, PB (prepared in $68 \%$ PG and $10 \% \mathrm{EtOH}$ ) is administered intravenously at a dose of up to $40 \mathrm{mg} / \mathrm{kg}$ over a brief period of time (usually $<1 \mathrm{~h}$ ). Unfortunately, at least $50 \%$ of patients are still seizing after receiving the lorazepam/PB regimen. For these patients, phenytoin (an anticonvulsant that is also dissolved in PG and EtOH), fosphenytoin (an anticonvulsant that is not dissolved in PG or EtOH), or additional PB is given until seizures are arrested. Since unmitigated seizure activity results in widespread excitotoxic damage in the brain, our findings do not suggest that clinicians should not aggressively treat status epileticus but rather suggest that treatments utilizing a nontoxic solvent are needed.

PG is not given in isolation but is typically used in conjunction with another agent. To determine the true effect of using PG as a solvent, we investigated whether a mixture of PB dissolved in PG could produce more neuroapoptosis than PB dissolved in saline. Individual agents were used at doses that alone do not produce apoptosis: $5 \mathrm{mg} / \mathrm{kg}$ PB (standard clinical maintenance dose) and 68\% PG (0.68 ml/kg; Figure 4). Our results indicate that $\mathrm{PB}$ dissolved in PG produces significantly greater apoptosis than PB dissolved in saline, raising additional concerns about the safety of PG when used as a solvent. In these cases in which mixtures of compounds are used, the toxic threshold dose of PG would be lower than $1-2 \mathrm{ml} / \mathrm{kg}$. Assessing the combined effect of chemicals is complex and often unpredictable (28). Potential effects include additivity, in which agents are no more and no less effective in combination than when they are separate, and synergism, in which the effectiveness of agents is increased when in combination (29). It is uncertain whether the observed effects in our study represent synergism or additivity. However, it is clear that the combination of two agents at nontoxic doses can lead to a significant toxic effect. To differentiate between these two effects, more rigorous testing is required such as the "dose additivity" and "effect additivity" methods of analyzing interactions (28).

In human infants, $\mathrm{PG}$ exposure has been associated with many acute toxic effects including CNS disturbances such as drowsiness and confusion $(9,10,12,30,31)$. Rigorous behavioral experiments were not conducted in our study, but we noticed that sedation and drowsiness were seen in animals treated with higher PG doses. However, at lower doses in which significant apoptosis was still detectable, there was no notable drowsiness. This finding suggests that apoptosis could occur in humans who have accumulated levels of PG that are high enough to develop acute CNS disturbances. More importantly, it is still unknown whether apoptosis results in long-term cognitive and behavioral abnormalities. This issue for PG has not been addressed in either humans or animals. For EtOH, a single several-hour exposure in P7 mice results in substantial apoptosis and produces detectable impairments in spatial learning and memory when animals are tested as juveniles (32). Furthermore, EtOH-induced apoptosis is considered to be a potential mechanism accounting for some of the CNS sequelae associated with fetal alcohol syndrome in humans (33). Further studies are needed to clarify the longterm CNS consequences of PG exposure, including potential cognitive and behavioral outcomes.

\section{METHODS}

\section{Animals and Drugs}

Research procedures were approved by the Institutional Animal Care and Use Committee of Washington University in St Louis. C57BL/6 mice (Harlan, Indianapolis, IN) were used in all experiments. For single-dose exposure studies, animals received a single i.p. injection of $100 \%$ (v/v) PG at various doses (1-10 $\mathrm{ml} / \mathrm{kg}$; laboratory grade (USP/FCC; $>99 \%$ pure); Fisher Scientific, Fair Lawn, NJ) or $10 \mathrm{ml} / \mathrm{kg}$ body weight of saline. To detect apoptotic cell death in the developing brain, P7 mice $(n=12)$ were exposed to a one-time 10-ml $/ \mathrm{kg}$ dose of PG. To determine the doseresponse effects, P7 mice $(n=36)$ were exposed to various doses of PG $(0,1,2,3,5$, and $10 \mathrm{ml} / \mathrm{kg})$. To determine whether animals of different ages are sensitive to PG-induced apoptosis, P4, P7, P14, P17, P24, and P30 mice ( $n=72)$ were exposed to a one-time $10 \mathrm{ml} / \mathrm{kg}$ dose of PG. To determine whether PG would augment the apoptotic potential of PB, 
combination studies were conducted. P7 mice $(n=26)$ were exposed to a one-time dose of PB (5 mg/kg; 5-ethyl-5-phenyl-2,4,6-trioxohexahydropyrimidine; Sigma-Aldrich, St. Louis, MO), which was prepared as a solution dissolved in either saline or $68 \%(\mathrm{v} / \mathrm{v})$ PG $(0.68 \mathrm{ml} / \mathrm{kg}$ administered intraperitoneally). The $5-\mathrm{mg} / \mathrm{kg}$ dose of $\mathrm{PB}$ was previously determined to be nontoxic (i.e., exposure does not increase apoptosis when compared to saline). Treatment conditions were distributed equally between sexes and in preweaning animals across litters in order to control for potential litter or gender effects. At various intervals between 2 and $24 \mathrm{~h}$ following treatment, animals were anesthetized with sodium pentobarbital and perfused as previously described (4). Brains were processed with one of three histological procedures.

\section{Histological Procedures}

For AC-3 immunohistochemistry and de Olmos silver staining, brains were cut into $70-\mu \mathrm{m}$ transverse sections on a vibratome. Sections were processed according to protocols previously described (4). Staining was visualized with light microscopy. Areas of damage were identified using an atlas of the developing mouse brain (34). Plastic sections for electron microscopy were prepared as previously described (4) and viewed in a JEOL 100 CX transmission electron microscope (Peabody, MA).

\section{Stereology}

Cell counts were performed using Stereoinvestigator 7.5 (MicroBrightField, Colchester, VT). Every eighth section was chosen from each brain (excluding brainstem and cerebellum) and processed for AC-3 immunohistochemistry. The optical fractionator technique was used to obtain unbiased estimates of the total number of apoptotic cells in the brain. Guard volume was set to $5.0 \mu \mathrm{m}$, and intersection interval, counting frame size, and distance between counting frames were adjusted to sample approximately 200 degenerating cells. The counter was blinded to litter, gender, and treatment.

\section{Statistical Analysis}

Data are presented as mean $( \pm$ SEM). Dose-response analysis data were evaluated using a four-parameter logistic equation (GraphPad Prism Software, San Diego, CA). A one-way ANOVA with subsequent post hoc comparisons was used to judge significance of the observed effect. For mixture effect studies, comparison of data was calculated by Student's two-tailed $t$-test following tests to determine normality of data. Differences were considered significant at $P<0.05$.

\section{STATEMENT OF FINANCIAL SUPPORT}

This work was supported by the National Institutes of Health (P30HD062171, R01 ES012443 to N.B.F., and K01MH083046 to K.K.N.).

\section{REFERENCES}

1. Lockshin RA, Zakeri Z. Programmed cell death and apoptosis: origins of the theory. Nat Rev Mol Cell Biol 2001;2:545-50.

2. Ikonomidou C, Bittigau P, Ishimaru MJ, et al. Ethanol-induced apoptotic neurodegeneration and fetal alcohol syndrome. Science 2000;287:1056-60.

3. Olney JW, Tenkova T, Dikranian K, et al. Ethanol-induced caspase-3 activation in the in vivo developing mouse brain. Neurobiol Dis 2002; 9:205-19.

4. Hanslick JL, Lau K, Noguchi KK, et al. Dimethyl sulfoxide (DMSO) produces widespread apoptosis in the developing central nervous system. Neurobiol Dis 2009;34:1-10.
5. Dobbing J, Sands J. Comparative aspects of the brain growth spurt. Early Hum Dev 1979;3:79-83.

6. Food and Drug Administration. GRAS status of propylene glycol and propylene glycol monostearate. Fed Regist 47:27810. 21 CFR 184.1666, 1982.

7. Joint FAO/WHO Expert Committee on Food Additives (JECFA). Toxicological evaluation of some food additives including anticaking agents, antimicrobials, antioxidants, emulsifiers and thickening agents. WHO Food Additives Series, No. 5, Geneva, 1974.

8. American Academy of Pediatrics. "Inactive" ingredients in pharmaceutical products Committee on Drugs. Pediatrics 1985;76:635-43.

9. Martin G, Finberg L. Propylene glycol: a potentially toxic vehicle in liquid dosage form. J Pediatr 1970;77:877-8.

10. Arulanantham K, Genel M. Central nervous system toxicity associated with ingestion of propylene glycol. J Pediatr 1978;93:515-6.

11. Demey HE, Daelemans RA, Verpooten GA, et al. Propylene glycol-induced side effects during intravenous nitroglycerin therapy. Intensive Care Med 1988;14:221-6.

12. Guillot M, Bocquet G, Eckart P, et al. [Home environment and acute propylene glycol intoxication in a two-year old. An unusual case report]. Arch Pediatr 2002;9:382-4.

13. Yaucher NE, Fish JT, Smith HW, Wells JA. Propylene glycol-associated renal toxicity from lorazepam infusion. Pharmacotherapy 2003;23:1094-9.

14. Wilson KC, Reardon C, Farber HW. Propylene glycol toxicity in a patient receiving intravenous diazepam. N Engl J Med 2000;343:815.

15. Wilson KC, Reardon C, Theodore AC, Farber HW. Propylene glycol toxicity: a severe iatrogenic illness in ICU patients receiving IV benzodiazepines: a case series and prospective, observational pilot study. Chest 2005;128: 1674-81.

16. Bledsoe KA, Kramer AH. Propylene glycol toxicity complicating use of barbiturate coma. Neurocrit Care 2008;9:122-4.

17. Miller MA, Forni A, Yogaratnam D. Propylene glycol-induced lactic acidosis in a patient receiving continuous infusion pentobarbital. Ann Pharmacother 2008;42:1502-6.

18. Food and Drug Administration. March 8. Drug Safety Communication: Serious health problems seen in premature babies given Kaletra (lopinavir/ritonavir) oral solution, 2011. (http://www.fda.gov/Drugs/DrugSafety/ ucm246002.htm\#safety_announcement.)

19. Chicella M, Jansen P, Parthiban A, et al. Propylene glycol accumulation associated with continuous infusion of lorazepam in pediatric intensive care patients. Crit Care Med 2002;30:2752-6.

20. Shehab N, Lewis CL, Streetman DD, Donn SM. Exposure to the pharmaceutical excipients benzyl alcohol and propylene glycol among critically ill neonates. Pediatr Crit Care Med 2009;10:256-9.

21. Bittigau P, Sifringer M, Genz K, et al. Antiepileptic drugs and apoptotic neurodegeneration in the developing brain. Proc Natl Acad Sci USA 2002;99:15089-94.

22. Farber NB, Olney JW. Drugs of abuse that cause developing neurons to commit suicide. Brain Res Dev Brain Res 2003;147:37-45.

23. Kuan CY, Roth KA, Flavell RA, Rakic P. Mechanisms of programmed cell death in the developing brain. Trends Neurosci 2000;23:291-7.

24. Kerr JF, Wyllie AH, Currie AR. Apoptosis: a basic biological phenomenon with wide-ranging implications in tissue kinetics. Br J Cancer 1972;26:239-57.

25. Ishimaru MJ, Ikonomidou C, Tenkova TI, et al. Distinguishing excitotoxic from apoptotic neurodegeneration in the developing rat brain. J Comp Neurol 1999;408:461-76.

26. Lu C, Mattson MP. Dimethyl sulfoxide suppresses NMDA- and AMPAinduced ion currents and calcium influx and protects against excitotoxic death in hippocampal neurons. Exp Neurol 2001;170:180-5.

27. Ikonomidou C, Bosch F, Miksa M, et al. Blockade of NMDA receptors and apoptotic neurodegeneration in the developing brain. Science 1999;283:70-4.

28. Lau K, McLean WG, Williams DP, Howard CV. Synergistic interactions between commonly used food additives in a developmental neurotoxicity test. Toxicol Sci 2006;90:178-87.

29. Berenbaum MC. What is synergy? Pharmacol Rev 1989;41:93-141. 


\section{Articles | Lau et al.}

30. Glasgow AM, Boeckx RL, Miller MK, MacDonald MG, August GP, Goodman SI. Hyperosmolality in small infants due to propylene glycol. Pediatrics 1983;72:353-5.

31. Fligner CL, Jack R, Twiggs GA, Raisys VA. Hyperosmolality induced by propylene glycol. A complication of silver sulfadiazine therapy. JAMA 1985;253:1606-9.

32. Wozniak DF, Hartman RE, Boyle MP, et al. Apoptotic neurodegeneration induced by ethanol in neonatal mice is associated with profound learning/ memory deficits in juveniles followed by progressive functional recovery in adults. Neurobiol Dis 2004;17:403-14.

33. Olney JW, Wozniak DF, Farber NB, Jevtovic-Todorovic V, Bittigau P, Ikonomidou C. The enigma of fetal alcohol neurotoxicity. Ann Med 2002;34:109-19.

34. Paxinos G, Halliday G, Watson C, Koutcherov Y, Wang H. Atlas of the Developing Mouse Brain. Amsterdam, The Netherlands Elsevier, 2007. 